\title{
Estimation of Viscoelastic Properties of Cells Using Acoustic Tweezing Cytometry
}

\author{
Chunmei Yang, PhD, Di Chen, PhD, Xiaowei Hong, PhD
}

Received November 2, 2015, from the Department of Biomedical Engineering, Tianjin University, Tianjin, China (C.Y.); and Department of Biomedical Engineering, University of Michigan, Ann Arbor, Michigan USA (C.Y., D.C., X.H.). Revision requested January 13, 2016. Revised manuscript accepted for publication March 4, 2016.

We thank Cheri X. Deng, $P h D$, for valuable comments and suggestions that greatly enhanced the manuscript. Dr Yang acknowledges funding support from the China Scholarship Council and the Natural Science Foundation of Tianjin (grant 12JCYBJC18300).

Address correspondence to Chunmei Yang, $\mathrm{PhD}$, Department of Biomedical Engineering, Tianjin University, 92 Weijin Rd, Building 17, No. 223, 300072 Tianjin, China.

E-mail:yangcm@tju.edu.cn

Abbreviations

RDG, Arg-Gly-Asp

doi:10.7863/ultra.15.10076
Objectives - Recently developed acoustic tweezing cytometry uses ultrasound-responsive targeted microbubbles for biomechanical stimulation of live cells at the subcellular level. The purpose of this research was to estimate the viscoelastic characteristics of cells from the displacements of cell-bound microbubbles in response to ultrasound pulses on acoustic tweezing cytometry.

Methods-Microbubbles were bound to NIH/3T3 fibroblasts and ATDC5 cells through an integrin-cytoskeleton linkage. The evolution of microbubble behaviors under irradiation by ultrasound pulses was captured by a high-speed camera and tracked by a customized algorithm. The total damping constant, stiffness, and rigidity of the cells were estimated by fitting the measured temporal displacement profiles to a KelvinVoigt-based model.

Results - The mean maximum displacement of the microbubbles attached to $\mathrm{NIH} / 3 \mathrm{~T} 3$ fibroblasts was much greater than that for ATDC5 cells. The mean fitted damping constant and stiffness \pm SD for ATDC5 cells were $28.16 \pm 7.08 \mathrm{mg} / \mathrm{s}$ and $0.5041 \pm 0.1381 \mathrm{mN} / \mathrm{m}$, respectively, and the values for $\mathrm{NIH} / 3 \mathrm{~T} 3$ fibroblasts were $13.12 \pm 4.23 \mathrm{mg} / \mathrm{s}$ and $0.2591 \pm 0.0715 \mathrm{mN} / \mathrm{m}$. The rigidity for ATDC5 cells was $331.46 \pm 106.50 \mathrm{MPa}$, whereas that for $\mathrm{NIH} / 3 \mathrm{~T} 3$ fibroblasts was $117.92 \pm 34.83 \mathrm{MPa}$.

Conclusions - The Arg-Gly-Asp-integrin-cytoskeleton system of NIH/3T3 fibroblasts appears to be softer than that of ATDC5 cells. The rigidity of ATDC5 cells was significantly greater than that of NIH/3T3 fibroblasts at the 95\% confidence level. This strategy provides a novel way to determine the viscoelastic properties of the live cells.

Key Words — acoustic tweezing cytometry; cell viscoelasticity; microbubble; ultrasound

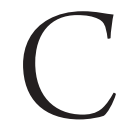

ellular viscoelasticity is a key factor in regulating the behavior of cells, such as cell growth, stem cell differentiation, cell crawling, wound healing, protein regulation, cell malignancy, and even apoptosis. ${ }^{1,2}$ Single-cell mechanical properties were found to be related to gene and protein expression and can be used to distinguish differences in cellular subpopulations, disease state, and tissue sources. ${ }^{3}$ Recent studies suggested that cellular mechanical properties (including stiffness and viscoelastic properties) as effective "mechanical biomarkers" could be used to determine specific phenotypic characteristics of stem cells and predict their differentiation capability. ${ }^{3-6}$

Targeted microbubbles that can be bound to specific receptors on the cell membrane have been exploited as multifunctional agents for intracellular drug delivery and biomechanical stimulation of mechanosensitive cells. ${ }^{78}$ Ultrasound-induced bubble activities can 
induce substantial mechanical impacts on cells, such as sonoporation for gene and drug delivery. ${ }^{7,9}$ Oscillatory positive and negative pressure of an ultrasound field can induce bubble expansion and contraction or violent collapse if the pressure amplitude is high enough. ${ }^{10-12} \mathrm{~A}$ long ultrasound pulse with a low amplitude can generate a directional acoustic radiation force on the bubble, ${ }^{13}$ which can compress the bubble against the cell membrane to exert a mechanical force on the cell.

Recently, Fan et $\mathrm{al}^{7}$ reported a novel acoustic tweezing cytometric technique that used ultrasound excitation of membrane-bound gaseous microbubbles to generate controllable subcellular mechanical stimulations in live single cells. Using NIH/3T3 fibroblasts and human mesenchymal stem cells as mechanosensitive cell models, they showed that ultrasound excitation of lipid microbubbles attached to the cell membrane through integrin-mediated adhesions could elicit a rapid and sustained reactive intracellular cytoskeleton contractile force increase. In addition, they showed that the total displacement of a microbubble caused by acoustic radiation force during the ultrasound application period appeared to be positively correlated with the overall cytoskeleton contractile force increase.

Yang and Church ${ }^{14}$ developed a model to study the dynamics of bubbles in a sound field with soft tissue. In this model, they combined the Keller-Miksis equation with the linear Kelvin-Voigt model for large-amplitude oscillations of bubbles in a viscoelastic medium. The model incorporated experimental data on viscoelastic properties of soft tissue at megahertz frequencies and was better suited than the Rayleigh-Plesset equation to simulate large-amplitude bubble oscillations. ${ }^{14}$ In this work, we proposed to estimate the viscoelastic properties of cells from the displacements of targeted microbubbles by application of acoustic tweezing cytometry using this model. By fitting the temporal displacement profile of the bound microbubbles in response to ultrasound pulses, we were able to estimate the total damping constant, stiffness, and rigidity of different cell types.

\section{Materials and Methods}

\section{Cell Culture and Targeted Microbubbles}

The ATDC5 cell line (Sigma Aldrich, St Louis, MO) and NIH/3T3 mouse fibroblast line (American Type Culture Collection, Manassas, VA) were used in this study. ATDC5 cells were maintained in flasks in complete growth medium consisting of Dulbecco's modified Eagle's/F12 medium (Gibco, Grand Island, NY) supplemented with $10 \%$ fetal bovine serum and $1 \%$ penicillin-streptomycin at $37^{\circ} \mathrm{C}$ and $5 \%$ carbon dioxide. $\mathrm{NIH} / 3 \mathrm{~T} 3$ fibroblasts were maintained in flasks in growth medium consisting of low-glucose Dulbecco's modified Eagle's medium supplemented with $10 \%$ bovine serum (Invitrogen, Carlsbad, CA), $1 \% \mathrm{~L}$-glutamine, and $1 \%$ penicillin/streptomycin at $37^{\circ} \mathrm{C}$ and $5 \%$ carbon dioxide. Cells were plated in a fibronectincoated glass-bottom 35-mm Petri dish for 1 day to reach $70 \%$ confluency before the experiments. Targesphere-SA microbubbles (Targeson, La Jolla, CA) were used in this study and bound to the integrin of the cells. The microbubbles were first mixed with biotinylated Arg-Gly-Asp (RGD) molecules (Peptides International, Louisville, KY) for 20 minutes at room temperature, with a volume ratio of 5:1 between microbubbles $\left(3 \times 10^{9} / \mathrm{mL}\right)$ and RGD $(2 \mathrm{mg} / \mathrm{mL})$ to form RGD microbubbles. To conjugate the microbubbles with cells, the culture medium from the cell-seeded dish was removed, followed by immediate addition of 20 $\mu \mathrm{L}$ of the diluted RGD microbubbles $\left(3 \times 10^{7} / \mathrm{mL}\right)$. Then the dish was flipped over so that the attached cells faced downward to facilitate attachment of the microbubbles with the cells via RGD-integrin binding. After 10 minutes, the dish was flipped back and gently washed with complete culture medium to remove unbound RGD microbubbles before experiments.

\section{Experimental Setup and Ultrasound System}

As described previously, ${ }^{8}$ the $35-\mathrm{mm}$ Petri dish with cells and targeted microbubbles was placed on the stage of an inverted microscope (Eclipse Ti-U; Nikon, Melville, NY). A single-element planar transducer $(5 \mathrm{MHz}$; Advanced Devices, Wakefield, MA), driven by a waveform generator (33250A; Agilent Technologies, Palo Alto, CA) and a 75-W power amplifier (75A250; Amplifier Research, Souderton, $\mathrm{PA}$ ), was used to generate single ultrasound pulses with a peak negative pressure of $0.03 \mathrm{MPa}$ and pulse duration of 500 milliseconds. The transducer was mounted at a $45^{\circ}$ angle with its active surface submerged in medium and 11.5 $\mathrm{mm}$ from the cells to avoid standing waves in the dish.

\section{High-Speed Video Microscopy of Ultrasound-Induced Bubble Activities}

Low-amplitude ultrasound pulses with a peak negative pressure of $0.03 \mathrm{MPa}$, center frequency of $5 \mathrm{MHz}$, and pulse duration of 0.5 seconds were applied to individual $\mathrm{NIH} / 3 \mathrm{~T} 3$ fibroblasts and ATDC5 cells with one microbubble attached to the cell membrane. To capture the evolution of microbubble behaviors driven by ultrasound pulses, we used a high-speed camera (FASTCAM SA1; Photron, San Diego, CA) with a frame rate of 1000 frames per second. A customized MATLAB algorithm (The MathWorks, Natick, MA) was used to track microbubbles. The dis- 
placement of a microbubble (1.8-2.2 $\mu \mathrm{m}$ in radius) from its original location as a function of time was obtained from the collected image sequences.

\section{Fitting of Bubble Displacements and Estimation of the Viscoelastic Properties of Cells}

A cell-attached microbubble exposed to an ultrasound field will be subjected to a number of forces, including the applied acoustic pressure and restoring forces due to the RGDintegrin-cytoskeleton linkage. The model proposed by Yang and $\mathrm{Church}^{14}$ for viscoelastic medium is used to consider the dynamics of bubbles in this RGD-integrincytoskeleton system. Basically, the dynamics of a spherical bubble in an unbound viscoelastic medium is described by

$$
\begin{aligned}
& \left(1-\frac{\dot{R}}{c}\right) \rho R \ddot{R}+\frac{3}{2}\left(1-\frac{\dot{R}}{3 c}\right) \rho \dot{R}^{2}= \\
& \left(1+\frac{R}{c}\right)\left(p_{a}-p_{I}\right)+\frac{R}{c} \frac{d}{d t}\left(p_{a}-p_{I}\right),
\end{aligned}
$$

with

$$
\begin{aligned}
& p_{a}=p_{g}-\frac{2 \sigma}{R}+\tau_{r r}(R, t), p_{a}-p_{I}= \\
& p_{g}-\frac{2 \sigma}{R}-p_{0}+p_{A}+3 \int_{R}^{\infty} \frac{\tau_{r r}}{r} d r,
\end{aligned}
$$

where $R$ is the position of the gas-tissue interface; the dot indicates the time derivative, $\rho$ is the density; $c$ is the sound speed in the medium; $p_{a}$ is the pressure at the bubble surface; $p_{I}$ is the pressure at infinity; $p_{\mathrm{g}}$ is the gas pressure inside the bubble; $\sigma$ is the surface tension; $p_{0}$ is the static pressure; $p_{A}$ is the driving pressure; $r$ is the radial coordinate; and $\tau_{r r}$ is the stress in $\mathrm{r}$ direction.

The combination of the Kelvin-Voigt model to account for the elasticity of soft tissue $\left(p_{a}-p_{I}\right)$ can be expanded as

$$
p_{a}-p_{I}=p_{g}-\frac{2 \sigma}{R}-p_{0}+p_{A}-\left[\frac{4 G}{3 R}\left(R^{3}-R_{0}^{3}\right)+\frac{4 \mu \dot{R}}{R}\right],
$$

where $R_{0}$ is the bubble equilibrium radius; $G$ is the shear modulus (or rigidity); and $\mu$ is the viscosity of the medium.

At a low-pressure amplitude, the pulsation amplitude $x=R-R_{0}$ is assumed to be small. Under linear approximation, Equation 1 may be rewritten as ${ }^{10,14,15}$

$$
m \ddot{x}+b \dot{x}+k x=F_{d},
$$

where $m=4 \pi R_{0}^{3} \rho$ is the effective mass of a bubble; $b$ is the total damping from the radiation, medium, and bubble- integrin-cytoskeleton system; and $k$ is the stiffness. $F_{d}$ is the acoustic radiation force and is computed as ${ }^{8}$

$$
F_{d}=\frac{4 \pi \beta \omega^{2} R_{0}\left(P_{u}\right)^{2}}{\rho c\left[\left(\omega_{0}^{2}-\omega^{2}\right)^{2}+4 \beta^{2} \omega^{2}\right]},
$$

with $^{16}$

(6)

$$
\omega_{0}=\sqrt{\frac{3 \kappa p_{0}}{\rho R_{0}{ }^{2}}+\frac{2 s_{p}}{\rho R_{0}^{3}}}, \beta=\left(\frac{\omega R_{0}}{c}+\frac{4 \mu}{\omega \rho R_{0}{ }^{2}}+\frac{s_{f}}{4 \pi \omega \rho R_{0}^{3}}\right) \omega_{0} / 2,
$$

where $K$ is the ploytropic index of bubble gas; $s_{p}$ is the stiffness of the bubble shell; $\mu$ is the viscosity of the medium; sf is the friction of the bubble shell; $P_{u}$ is the applied pressure amplitude; and $\omega=2 \pi f$ is the angular frequency of the applied ultrasound.

In this work, we fitted Equation 4 to the measured displacements of the bound microbubbles using the MAT$\mathrm{LAB}$ ordinary differential equation solver to estimate the total damping constant $b$ and stiffness $k$ of the bubbleRGD-integrin-cytoskeleton system.

Five components contribute to the total damping: viscous, thermal, acoustic, interfacial, and elastic, which can be expressed as ${ }^{14,15}$

$$
b=b_{v i s}+b_{t h}+b_{a c}+b_{\text {int }}+b_{\text {ela }} .
$$

According to the modeling of Yang and Church, ${ }^{14}$ for frequencies of greater than $1 \mathrm{MHz}$ and radii of less than the resonance radius of the bubble, the contribution of the thermal term $b_{\text {th }}$ and interfacial term $b_{\text {int }}$ to the total damping is trivial and can be neglected. Therefore, we only considered the other 3 terms: viscous component $\left(b_{v i s}\right)$, acoustic component $\left(b_{a c}\right)$, and elastic component $\left(b_{\text {ela }}\right)$ here.

Considering a bubble interacting with a viscoelastic tissue, the viscous, acoustic, and elastic components can be accordingly expressed as ${ }^{14}$

$$
\begin{aligned}
& b_{v i s}=16 \pi R_{0} \mu, b_{a c}=\frac{4 \pi R_{0}^{2} \omega / c}{1+(\omega R / c)^{2}} \omega\left(\rho R_{0}{ }^{2}\right), \\
& b_{\text {ela }}=16 \pi R_{0}^{2} G / c .
\end{aligned}
$$

Therefore, we can estimate the shear modulus (rigidity) $G$ of the surrounding viscoelastic tissue from the elastic damping term $b_{\text {ela }}$. The initial conditions used in the fitting were $x_{0}=0$ and $x_{0}{ }^{\prime}=0$. The parameter values were $\rho=998$ $\mathrm{kg} / \mathrm{m}^{3}, c=1500 \mathrm{~m} / \mathrm{s}, \mu=0.007 \mathrm{~Pa} / \mathrm{s}, s_{p}=1.64 \mathrm{~N} / \mathrm{m}$, $s_{f}=0.15 \times 10^{-6} \mathrm{~kg} / \mathrm{s}, \kappa=1.06, P_{u}=0.03 \mathrm{MPa}$, and $f=5 \mathrm{MHz}$. 


\section{Results}

\section{Displacements of Cell-Bound Microbubbles on Acoustic Tweezing Cytometry}

When exposed to ultrasound pulses, the integrin-bound microbubble was pushed away from its original position by the acoustic radiation force during the "pulse-on" period. When the ultrasound was turned off, the microbubble was pulled back toward its original position due to the viscoelastic property of the bubble-RGD-integrin-cytoskeleton system. Examples of microbubbles attached to NIH/3T3 fibroblasts and ATDC5 cells and the changes in the radii and displacements of the microbubbles exposed to a single ultrasound pulse ( 0.5 second starting from 0 second) are illustrated in Figure 1.

During the experiments, we noted that the microbubbles attached to NIH/3T3 fibroblasts were displaced easier than the bubbles attached to ATDC5 cells under the same ultrasound parameters. The mean maximum displacement \pm SD of the microbubbles attached to NIH/3T3 fibroblasts $(1.12 \pm 1.07 \mu \mathrm{m} ; \mathrm{n}=62)$ was much greater than that for ATDC5 cells $(0.92 \pm 1.0 \mu \mathrm{m} ; n=79)$, indicating that the RGD-integrin-cytoskeleton system of NIH/3T3 fibroblasts appears to be softer than that of ATDC5 cells.

\section{Viscoelastic Properties of Cells From Model Fitting}

Examples of the fitting results are illustrated in Figure 2 for ATDC5 cells and NIH/3T3 fibroblasts. Statistical analyses of the damping constant and stiffness were performed for the representative data sets that had a maximum displacement of greater than $0.5 \mu \mathrm{m}$ and a coefficient of determination $\left(R^{2}\right)$ of greater than 0.8 . For ATDC5 cells, the mean of the fitted damping constant $(b)$ was $28.16 \pm 7.08 \mathrm{mg} / \mathrm{s}$, and stiffness $(k)$ was $0.5041 \pm 0.1381 \mathrm{mN} / \mathrm{m}(\mathrm{n}=64)$. For $\mathrm{NIH} / 3 \mathrm{~T} 3$ fibroblasts, the estimated damping constant was $13.12 \pm 4.23 \mathrm{mg} / \mathrm{s}$, and stiffness was $0.2591 \pm 0.0715 \mathrm{mN} / \mathrm{m}$ $(\mathrm{n}=52)$.

Based on the fitting of the representative experimental data, we calculated the rigidity of the cells. As a result, the estimated rigidity $(G)$ for ATDC5 cells was $331.46 \pm 106.50 \mathrm{MPa}$ $(\mathrm{n}=64)$, whereas that for $\mathrm{NIH} / 3 \mathrm{~T} 3$ fibroblasts was 117.92 $\pm 34.83 \mathrm{MPa}(\mathrm{n}=52)$. The difference in the rigidity between these cell types was significant at the $95 \%$ confidence level.

\section{Discussion}

In this work, we report a new strategy for determining the viscoelastic properties of live cells by using acoustic tweezing cytometry. By fitting the measured temporal displacement profiles of the microbubbles that were bound to the cells through integrin-cytoskeleton linkage under the irradiation of an ultrasound pulse, we obtained the total damping constant and stiffness for ATDC5 cells and NIH/3T3 fibroblasts. We further estimated the rigidity of the cells from the damping constant. It was found that the rigidity of ATDC5 cells was significantly greater than that of $\mathrm{NIH} / 3 \mathrm{~T} 3$ fibroblasts.

Cell viscoelasticity correlates with differentiation, aging, and disease states. Many events associated with stem cell differentiation during embryonic development and tissue regeneration are designed to change the cell shape and directly affect cytoskeleton tension and cell stiffness. ${ }^{17}$ It has become increasingly common to characterize and identify diseased and healthy cells by using cellular mechanical properties. ${ }^{18}$ As an alternative to identification by morphologic characteristics, cell stiffness has been proposed as a candidate for cancer cell detection. Atomic force microscopy is a popular tool for studying intrinsic cellular mechanical properties.

In a report on specifically determining the elastic and inelastic responses of single cells undergoing deformation with atomic force microscopy, Guolla et al ${ }^{19}$ quantified strain dynamics occurring along actin stress fibers throughout the cell directly and effects of cytoskeletal cross-linking on local deformation and strain dynamics. The Young modulus of actin stress fibers in NIH/3T3 fibroblasts was estimated to be $0.3 \mathrm{MPa}$, and the effective Young modulus of stress fibers in a living cell could be as high as $1 \mathrm{GPa}$ because of cross-linking with the rest of the cytoarchitecture. Considering the relationship between the Young modulus and the shear modulus, the rigidity estimated in this work should be reasonable.

In this work, the results were obtained by fitting the experimental data to the model developed by Yang and Church ${ }^{14}$ based on Keller-Miksis and Kelvin-Voigt models. It is a simplified linear model for providing analytical predictions of bubble responses to insonation with assumptions of incompressible media and ideal gas. As the wave equation describing ultrasound propagation in biological tissues is inherently nonlinear, there are inevitable errors in the linear approximation, especially for larger-amplitude oscillations. In addition, as the cell is located on one side of the bubble, whereas the culture medium is on the other, the bubble will be more strongly damped on one side than the other and therefore be distorted from sphericity. Even though we selected bubbles without detectable distortion for the analysis, distortion would undoubtedly affect the calculation of the acoustic radiation force and prediction of the model. This factor might in part cause large dispersion in the estimated damping constants as well as the stiffness of different microbubbles. Therefore, better prediction can 
be obtained with a more accurate model that accounts for bubble distortion and the nonlinearity of ultrasound propagation and viscoelastic media for large-amplitude oscillations. Furthermore, because of the complicated condition of the ultrasound field in the experimental dish, the acoustic radiation pressure finally applied on the microbubbles may not be ideally uniform. The dispersion in the radiation force should have caused the dispersion in the estimated

Figure 1. Examples of microbubbles bound to the cell membrane (left panel), the temporal change of the radius (middle panel), and displacement induced by a single ultrasound pulse of 0.5 seconds applied at 0 seconds (right panel).

(a) ATDC5

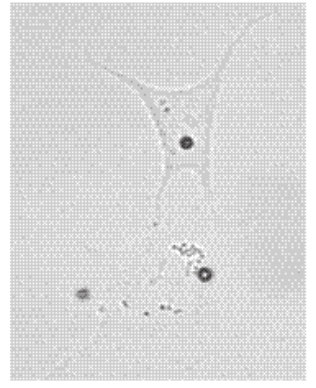

(b) $\mathrm{NIH} / 3 \mathrm{~T} 3$

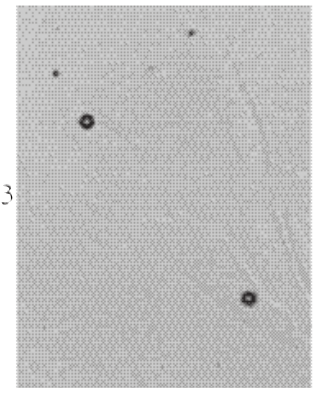

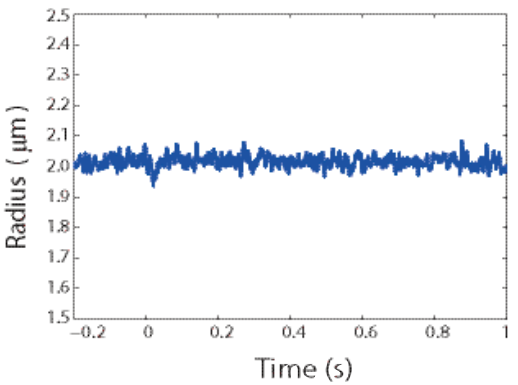
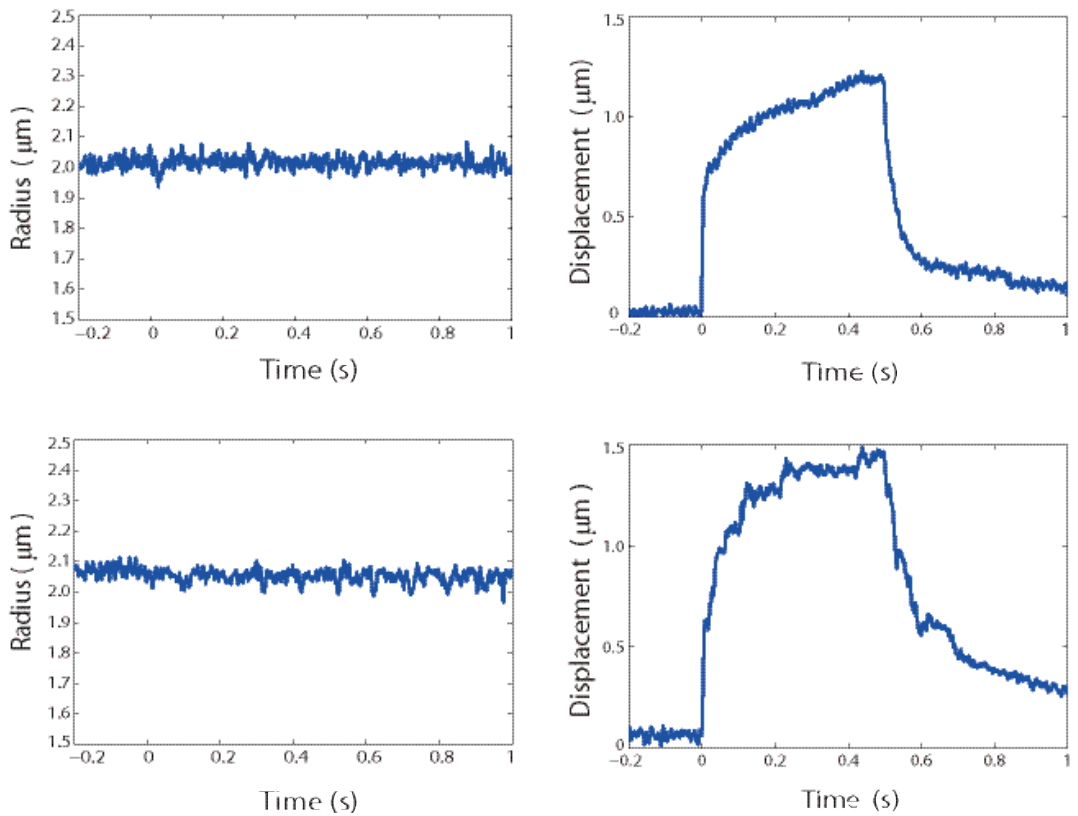

Figure 2. Example of fitting of the bubble displacements. The red plots indicate the measured displacements of microbubbles in response to single ultrasound pulse stimulation ( $0-0.5$ seconds), and the blue dashed plots indicate the fitted displacements with Equation 4.

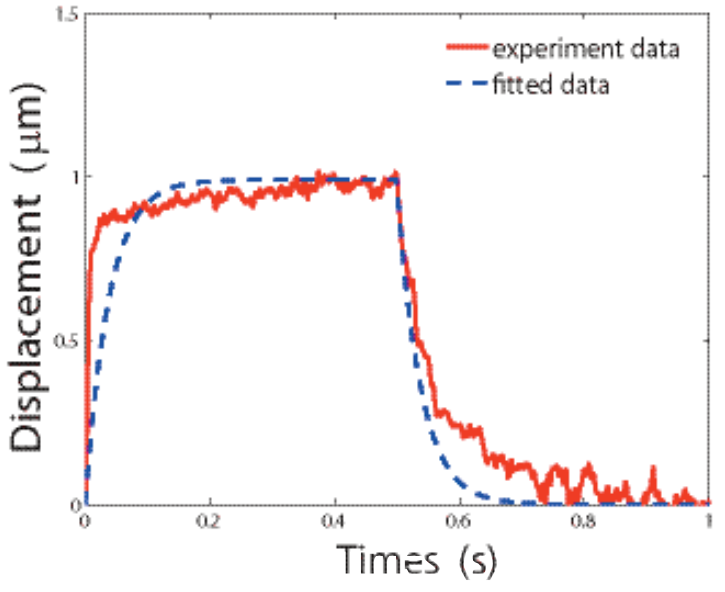

(a) ATDC5

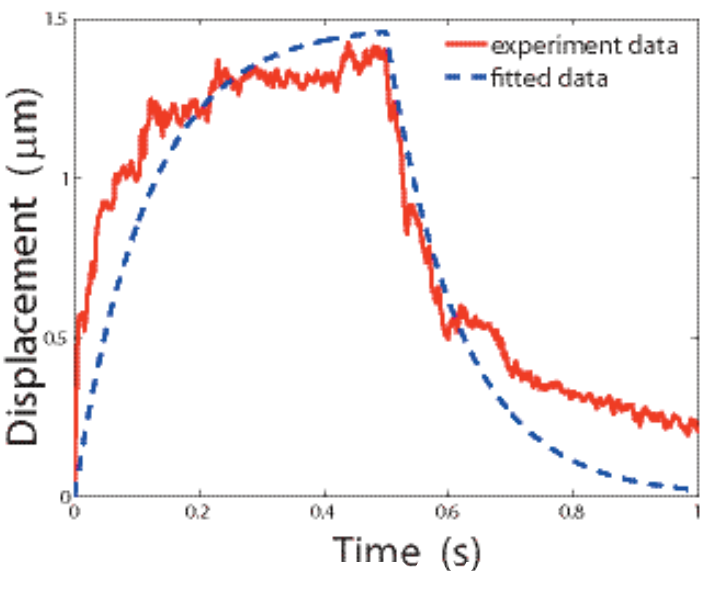

(b) $\mathrm{NIH} / 3 \mathrm{~T} 3$ 
parameters. A detailed measurement of the ultrasound field distribution in the dish can help greatly improve the estimation, which will be our future work. Otherwise, the modeling here was based on the displacements of microbubbles responding to a single ultrasound pulse. We can also model the displacements of microbubbles in response to multiple ultrasound pulses in future work.

In conclusion, this technique provides a versatile tool for cell rheometry by using acoustic tweezing cytometry. This method allows application of a controlled mechanical force at a subcellular level to a group of cells and simultaneous measurement of local viscoelastic properties by the use of targeted lipid microbubbles. Our results suggest that estimation of cell rheologic parameters using acoustic tweezing cytometry may provide a novel way to determine the viscoelastic properties of the live cells, which can be potentially used for cell sorting and screening.

\section{References}

1. Hoffman BD, Crocker JC. Cell mechanics: dissecting the physical responses of cells to force. Annu Rev Biomed Eng 2009; 11:259-288.

2. Janmey PA. The cytoskeleton and cell signaling: component localization and mechanical coupling. Physiol Rev 1998; 78:763-781.

3. González-Cruz RD, Fonseca VC, Darling EM. Cellular mechanical properties reflect the differentiation potential of adipose-derived mesenchymal stem cells. Proc Natl Acad Sci USA 2012; 109:E1523-E1529.

4. Darling EM, Topel M, Zauscher S, Vail TP, Guilak F. Viscoelastic properties of human mesenchymally-derived stem cells and primary osteoblasts, chondrocytes, and adipocytes. J Biomech 2008; 41:454-464.

5. Darling EM, Pritchett PE, Evans BA, Superfine R, Zauscher S, Guilak F. Mechanical properties and gene expression of chondrocytes on micropatterned substrates following dedifferentiation in monolayer. Cell Mol Bioeng 2009; 2:395-404.

6. Ekpenyong AE, Whyte G, Chalut K, et al. Viscoelastic properties of differentiating blood cells are fate- and function-dependent. PLoS One 2012; 7:e45237.

7. Fan Z, Sun Y, Chen D, et al. Acoustic tweezing cytometry for live-cell subcellular modulation of intracellular cytoskeleton contractility. Sci Rep 2013; 3:2176-2183

8. Chen D, Sun Y, Gudur MSR, et al. Two-bubble acoustic tweezing cytometry for biomechanical probing and stimulation of cells. Biophys J 2015; 108:32-42.

9. van Wamel A, Kooiman K, Harteveld M, et al. Vibrating microbubbles poking individual cells: drug transfer into cells via sonoporation. J Control Release 2006; 112:149-155.

10. Wu J, Nyborg WL. Ultrasound, cavitation bubbles and their interaction with cells. Adv Drug Deliv Rev 2008; 60:1103-1116.

11. O'Brien WD. Ultrasound-biophysics mechanisms. Prog Biophys Mol Biol 2007; 93:212-255
12. Miller DL. Overview of experimental studies of biological effects of medical ultrasound caused by gas body activation and inertial cavitation. Prog Biophys Mol Biol 2007; 93:314-330.

13. Dayton PA, Morgan KE, Klibanov AL, Brandenburger G, Nightingale KR, Ferrara KW. A preliminary evaluation of the effects of primary and secondary radiation forces on acoustic contrast agents. IEEE Trans Ultrason Ferroelectr Freq Control 1997; 59:1264-1277.

14. Yang X, Church CC. A model for the dynamics of gas bubbles in soft tissues. J Acoust Soc Am 2005; 118:3595-3606.

15. Prosperetti A. Thermal effects and damping mechanisms in the forced radial oscillations of gas bubbles in liquids. J Acoust Soc Am 1977; 61:1727.

16. Goertz DE, de Jong N, van der Steen AFW. Attenuation and size distribution measurements of Definity and manipulated Definity populations. Ultrasound Med Biol 2007; 33:1376-1388.

17. Sun Y, Chen CS, Fu J. Forcing stem cells to behave: a biophysical perspective of the cellular microenvironment. Annu Rev Biophys 2012; 41:519-542.

18. Haase K, Pelling AE. Investigating cell mechanics with atomic force microscopy. J R Soc Interface 2015; 12:20140970.

19. Guolla L, Bertrand M, Haase K, Pelling AE. Force transduction and strain dynamics in actin stress fibres in response to nanonewton forces. J Cell Sci 2012; 125:603-613 